\title{
INFINITE FROUDE NUMBER SOLUTIONS TO THE PROBLEM OF A SUBMERGED SOURCE OR SINK
}

\author{
G. C. HOCKING ${ }^{1}$
}

(Received 9 December 1986; revised 10 June 1987)

\begin{abstract}
The problem of a source or sink submerged beneath a free surface is investigated in the infinite Froude number limit. Solutions are found for all cases in which the source is situated away from the bottom of the channel. Solutions are also found for the case where the source is situated above the asymptotic level of the free surface, giving fountain type free surface shapes.
\end{abstract}

\section{Introduction}

Several recent investigations ([3], [4], [5], [9], [10]) have considered the free surface flow problem for the steady motion of an ideal fluid toward a line source or sink above a solid horizontal plane. One class of solutions to this problem gives a free surface shape in which a cusp exists above the sink (or source). These solutions can be related to the critical flow at which the fluid above an interface in a two-layer fluid will be drawn down into the sink if the flow rate is increased.

Two of these papers, Collings [3], and Yih [10], have discussed the linearised problem in which gravity approaches zero. Yih found the solution for the problem where the sink is situated on the bottom, while Collings found both this solution and another in which the sink is situated off the base. Yih also gave some solutions to the full nonlinear problem, for the problem where the base is not flat.

This paper presents solutions to the linearised problem for all cases where the sink is situated off the base, thus linking the solutions obtained by Collings and Yih. Solutions have also recently been found by Vanden-Broeck and Keller [9]

${ }^{1}$ Centre for Water Research, University of Western Australia, Nedlands, W.A. 6009, Australia

(C) Copyright Australian Mathematical Society 1988, Serial-fee code 0334-2700/88 
to the corresponding nonlinear problem, filling the gap in the work of Hocking [4].

It is shown that the resultant free-surface shapes are very much like those for the nonlinear problem [4], [9], [10] when the sink is on or near the base. The shape of the free surface changes to include a hump on the free surface for the situations in which the sink is higher off the bottom, similar to the nonlinear solutions of Vanden-Broeck and Keller [0]. Solutions exist in which the sink is located above the asymptotic level of the free surface at infinity.

\section{Integral equation and solution}

In this section, an integral equation is derived for the linearised problem. The resultant equation can be transformed to the airfoil equation [7] by removing that component of the solution which is known to satisfy the problem for the sink on the base, from Collings [3], and Yih [10].

The mathematical problem is to find a complex potential $w=\Phi+i \Psi$, which satisfies Laplace's equation within the flow domain, and which satisfies conditions of no flow across the solid boundaries and the free surface, and the condition of constant pressure on the free surface. This last condition is linearised by considering the limit as gravity tends to zero, reducing it to the condition of constant velocity on the free surface.

The transformation

$$
\exp (w)=\varsigma
$$

maps the infinite strip between $\Psi=0$ and $\Psi=-\pi$ in the $w$-plane to the lower half $\zeta$-plane. Without loss of generality we may choose to let $w=0$ correspond to the cusp point, so that the free surface $\Psi=0, \Phi>0$, lies along the real $\varsigma$-axis where $\varsigma \geq 1$. The sink lies at the origin in the $\varsigma$-plane, and the negative real axis corresponds to $\Psi=-\pi$ (see Figure 1).

We seek to find $w$ by solving for $\Omega(\varsigma)=\delta+i \tau$, defined in relation to the complex conjugate of the velocity field by

$$
w^{\prime}(z(\varsigma))=\exp (-i \Omega(\varsigma))
$$

The magnitude of the velocity at any point is then given by $\left|w^{\prime}(z)\right|=\exp (\tau(\varsigma))$, and the angle any streamline makes with the horizontal is $\delta(\varsigma)$. Thus for cusplike solutions, we require that $\delta=\pi / 2$ at $\varsigma=1$ and $\delta \rightarrow 0$ as $\varsigma \rightarrow \infty$. Note that we have nondimensionalised the problem with respect to the velocity of the flow at infinity, and the depth at infinity divided by $\pi$. The nondimensional flux from the source is $\pi$.

The free surface corresponds to the positive real $\varsigma$-axis for $\zeta>1$, and if we set $\tau=0$ in this region, the requirement of constant unit velocity is satisfied. On 


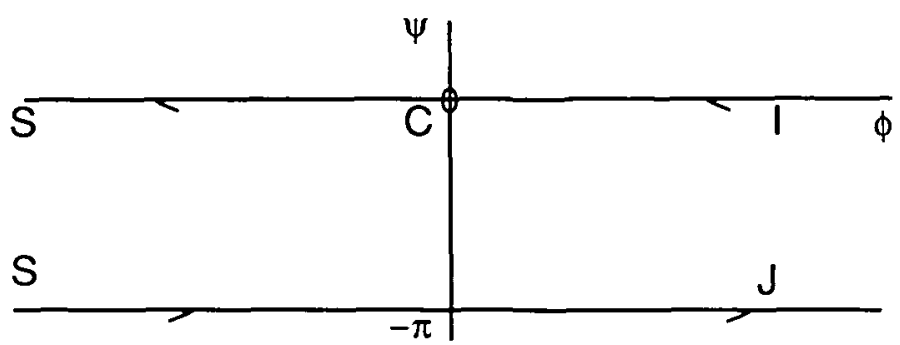

(a)

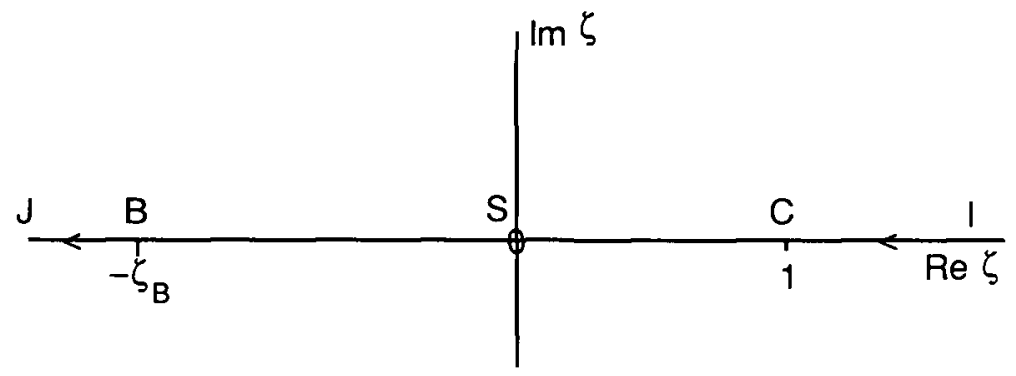

(b)

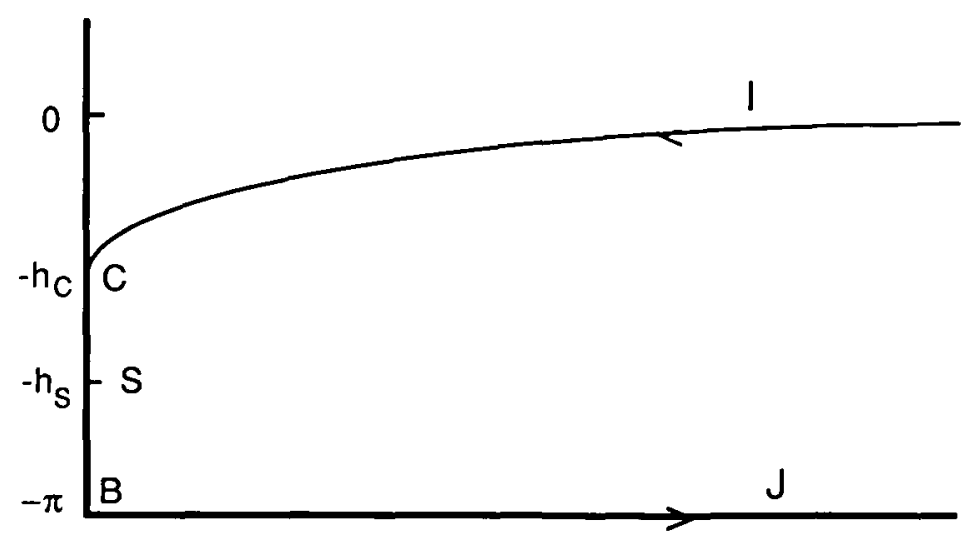

(c)

Figure 1. (a) The complex velocity potential $w$-plane,

(b) the lower half $\varsigma$-plane,

(c) the real z-plane. 
the remainder of the real $\varsigma$-axis, which corresponds to the solid boundaries of the flow domain, the streamlines must be parallel to the walls, so that if we choose $\delta$ to be the angle of the wall to the horizontal, that is, zero on $-\infty<\varsigma<\varsigma_{B}$, $-\pi / 2$ on $\zeta_{B}<\varsigma<0, \pi / 2$ on $0<\varsigma \leq 1$, then the conditions of no flow across the solid boundaries are satisfied.

The only singularities of the function $\Omega(\varsigma)$ in the $\zeta$-plane are those at the origin and at $\zeta=\zeta_{\bar{B}}$, corresponding to the sink and the stagnation point on the bottom beneath the sink respectively. Both of these singularities can be shown to be weaker than a simple pole, so that Cauchy's theorem can be applied to $\Omega(\varsigma)$ on a path consisting of the real $\varsigma$-axis and a semi-circle at $|\varsigma|=\infty$ in the lower half plane. Hence, for $\operatorname{Im}\{\zeta\}<0$ we have

$$
\Omega(\varsigma)=-\frac{1}{2 \pi i} \int_{-\infty}^{\infty} \frac{\Omega\left(\varsigma_{0}\right)}{\varsigma_{0}-\varsigma} d \varsigma_{0}
$$

since $\Omega \rightarrow 0$ as $|\zeta| \rightarrow \infty$. If we let $\operatorname{Im}\{\zeta\} \rightarrow 0^{-}$, we obtain

$$
\begin{aligned}
& \tau(\varsigma)=\frac{1}{\pi} \mathrm{CPV} \int_{-\infty}^{\infty} \frac{\delta\left(\varsigma_{0}\right)}{\varsigma_{0}-\varsigma} d \varsigma_{0}, \\
& \delta(\varsigma)=-\frac{1}{\pi} \mathrm{CPV} \int_{-\infty}^{\infty} \frac{\tau\left(\varsigma_{0}\right)}{\varsigma_{0}-\varsigma} d \varsigma_{0},
\end{aligned}
$$

where the integrals are of Cauchy principal value form.

Substituting the known values of $\delta(\zeta)$ into the equation for $\tau(\zeta)$ gives

$$
\tau(\varsigma)=\frac{1}{2} \log \left(\frac{(1-\varsigma)\left(\varsigma_{B}-\varsigma\right)}{\varsigma^{2}}\right)+\frac{1}{\pi} \mathrm{CPV} \int_{1}^{\infty} \frac{\delta\left(\varsigma_{0}\right)}{\varsigma_{0}-\varsigma} d \varsigma_{0}
$$

Setting $\tau=0$ on $\varsigma>1$ then gives an integral equation for $\delta(\varsigma)$ on $1<\varsigma<\infty$. If $\varsigma_{B}=0$, that is, if the sink is located on the base, the equivalent equation reduces to

$$
\frac{1}{2} \log \left(\frac{\varsigma-1}{\varsigma}\right)=-\frac{1}{\pi} \mathrm{CPV} \int_{1}^{\infty} \frac{\delta\left(\varsigma_{0}\right)}{\varsigma_{0}-\varsigma} d \varsigma_{0}
$$

to which the solution (Yih [10], Collings [3]) is

$$
\delta(\varsigma)=\arcsin \varsigma^{-1 / 2}
$$

If we let $\delta_{0}(\varsigma)$ be this solution for the case $\varsigma_{B}=0$, and write $\delta(\varsigma)$ as $\delta_{0}(\varsigma)+$ $\delta_{B}(\varsigma)$ in (2.5) then rearrange, we obtain

$$
\begin{aligned}
\frac{1}{2} \log & \left(\frac{\varsigma-1}{\varsigma}\right)+\frac{1}{2} \log \left(\frac{\varsigma-\varsigma_{B}}{\varsigma}\right) \\
= & -\frac{1}{\pi} \mathrm{CPV} \int_{1}^{\infty} \frac{\delta_{0}\left(\varsigma_{0}\right)}{\varsigma_{0}-\varsigma} d \varsigma_{0}-\frac{1}{\pi} \mathrm{CPV} \int_{1}^{\infty} \frac{\delta_{B}\left(\varsigma_{0}\right)}{\varsigma_{0}-\varsigma} d \varsigma_{0},
\end{aligned}
$$

on $1<\varsigma<\infty$. The first terms on each side of this equation, however, drop out since they are equal (from 2.6 and 2.7), leaving

$$
\frac{1}{2} \log \left(\frac{\varsigma-\varsigma_{B}}{\varsigma}\right)=-\frac{1}{\pi} \mathrm{CPV} \int_{1}^{\infty} \frac{\delta_{B}\left(\varsigma_{0}\right)}{\varsigma_{0}-\varsigma} d \varsigma_{0}
$$


a new integral equation for $\delta_{B}(\varsigma)$ along the real $\varsigma$-axis, $1<\varsigma<\infty$.

If we let $\zeta=(\xi+1 / 2)^{-1 / 2}$, equation (2.9) can be transformed to

$$
G\left(\varsigma_{B} ; \xi_{0}\right)=\frac{1}{\pi} \int_{-1 / 2}^{1 / \pi} \frac{F(\xi)}{\xi-\xi_{0}} d \xi
$$

where

$$
F(\xi)=\frac{\delta_{B}(\xi)}{\left(\xi+\frac{1}{2}\right)}
$$

and

$$
G\left(\varsigma_{B} ; \xi_{0}\right)=\frac{-1}{2\left(\xi_{0}+\frac{1}{2}\right)} \log \left(1-\varsigma_{B}\left(\xi_{0}+\frac{1}{2}\right)\right) .
$$

This equation is the airfoil equation [7], to which the solution is known [7], [6], and hence the solution to this problem can be written

$$
\delta_{B}(\xi)=\frac{1}{2 \pi} \xi^{1 / 2}(1-\xi)^{1 / 2} \int_{0}^{1} \frac{\log \left(1-\xi_{0} \zeta_{B}\right)}{\xi_{0}^{1 / 2}\left(1-\xi_{0}\right)\left(\xi-\xi_{0}\right)} d \xi_{0},
$$

where $\xi=\varsigma^{-1}$. Note that as $\zeta_{B}$ tends to zero, $\delta_{B}$ tends to zero and hence $\delta$ tends to $\delta_{0}$, the solution for the sink on the base. For this solution to be valid, $\delta_{B}$ must approach zero both as $\varsigma$ tends to one and as $\varsigma$ tends to infinity, and this is easily shown.

This integral has no closed-form solution, but can easily be solved numerically using a trapezoidal discretisation scheme, being sure to integrate through the singular point carefully. The solution can also be written as a series expansion in $\varsigma_{B}$ of the form

$$
\delta_{B}(\xi)=\frac{1}{2} \xi^{1 / 2}(1-\xi)^{1 / 2}\left(\varsigma_{B}+\frac{1}{2} \varsigma_{B}^{2}\left(\xi+\frac{1}{2}\right)+\frac{1}{3} \varsigma_{B}^{3}\left(\xi^{2}+\frac{1}{2} \xi+\frac{3}{8}\right)+O\left(\varsigma_{B}^{4}\right)\right),
$$

convergent for $-1 \leq \varsigma_{B} \leq 0$, and we can use this to test the numerical scheme.

Once $\delta_{B}$ is known, $\tau$ can be found from (2.5) and (2.7). Using $\delta$ and $\tau$, it is possible to integrate (2.2) to obtain the location of points on the free surface. These may be written as

$$
\begin{aligned}
& x(\varsigma)=x\left(\varsigma^{*}\right)+\int_{\varsigma^{*}}^{\varsigma} \varsigma^{-1} e^{-\tau(\varsigma)} \cos \delta(\varsigma) d \varsigma, \\
& y(\varsigma)=y\left(\varsigma^{*}\right)+\int_{\varsigma^{*}}^{\zeta} \varsigma^{-1} e^{-\tau(\varsigma)} \sin \delta(\varsigma) d \varsigma .
\end{aligned}
$$

Since $y \rightarrow 0$ as $\varsigma \rightarrow \infty$, the cusp depth is

$$
h_{C}=\int_{1}^{\infty} \varsigma^{-1} \sin \delta(\varsigma) d \varsigma,
$$

the sink depth is

$$
h_{S}=h_{C}+\int_{0}^{1} \varsigma^{-1} e^{-\tau(\varsigma)} d \varsigma
$$




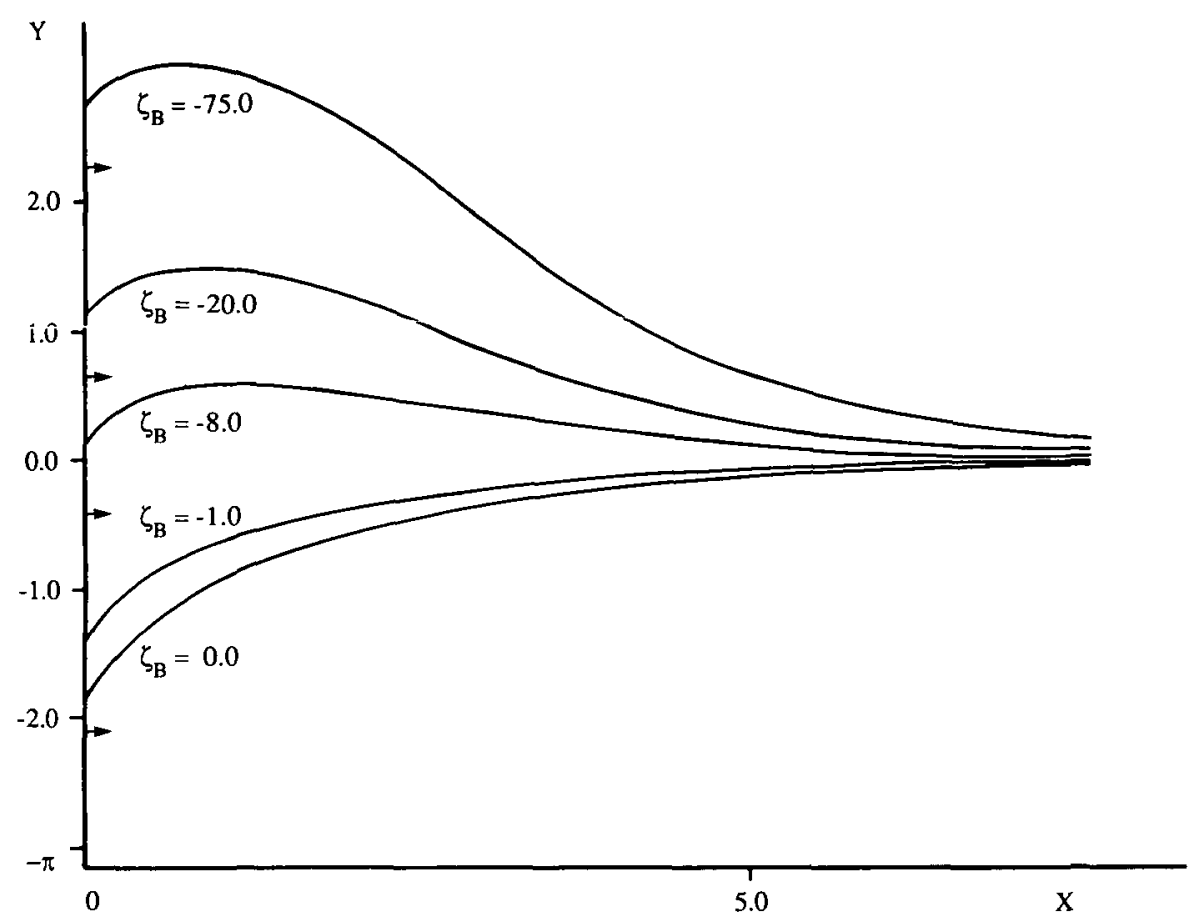

Figure 2. Some typical free-surface profiles.

and the base depth is

$$
h_{B}=h_{S}+\int_{0}^{\zeta B} \varsigma^{-1} e^{-\tau(\varsigma)} d \varsigma
$$

which must be equal to $\pi$ for all $\varsigma_{B}$, to conserve volume.

Substituting (2.7) and (2.12) into (2.14) gives a series expansion for $h_{C}$ in terms of $\zeta_{B}$, of the form

$$
h_{C} \sim 2+\frac{2}{3} \varsigma_{B}+\frac{1}{5} \varsigma_{B}^{2}+\frac{19}{210} \varsigma_{B}^{3}+\frac{95}{2024} \varsigma_{B}^{4}+O\left(\varsigma_{B}^{5}\right)
$$

Table 2.1 shows a comparison of the numerical solution to the series expansion for $h_{C}$, for values of $\left|\zeta_{B}\right|$ less than unity; the series does not converge for larger values of $\left|\varsigma_{B}\right|$. The series is not fully converged for the larger values shown, but the comparison verifies that the numerical scheme is performing adequately. 


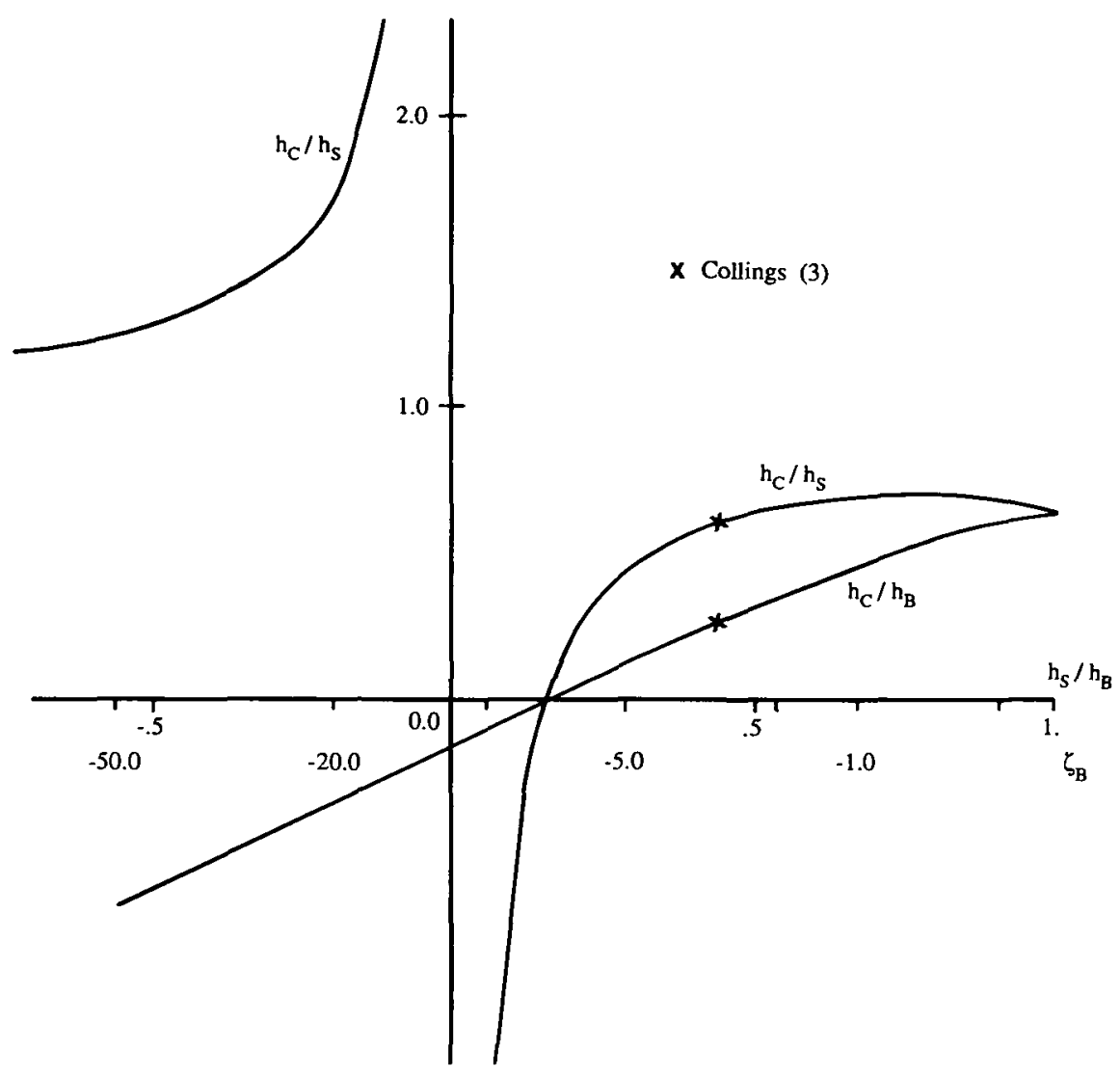

Figure 3. Plot of cusp to sink and cusp to base ratios against the sink to base depth ratio.

TABLE 2.1. Table showing the comparison of the series solution for the cusp depth to the numerical solution.

\begin{tabular}{|r|c|c|}
\hline \multirow{2}{*}{$\varsigma_{B}$} & \multicolumn{2}{|c|}{ Cusp Depth $h_{C}$} \\
\cline { 2 - 3 } & Series & Numerical \\
\hline 0.00 & 2.000 & 2.000 \\
-0.10 & 1.935 & 1.935 \\
-0.25 & 1.845 & 1.845 \\
-0.50 & 1.708 & 1.708 \\
-1.00 & 1.490 & 1.474 \\
\hline
\end{tabular}

Figure 2 shows typical free-surface shapes over a range of values of the sink depth. For $\left|\varsigma_{B}\right|$ small, the free surface rises monotonically from the cusp to the asymptotic level at infinity. When $\left|\zeta_{B}\right|$ is larger, the water rises to a maximum height before asymptoting to the level at upstream infinity from above. Solutions continue to exist even when the sink is situated above the level of the free surface 
at infinity. Figure 3 shows the cusp to sink and cusp to base depth ratios, plotted against the sink to base ratio. The results of Collings [3] and Yih [10] are shown to agree with those obtained here. In contrast to the findings of Collings, however, no particular fluid depth was found to be critical.

\section{Conclusions}

The solutions presented cover the full range of problems for a submerged sink (source) situated above a flat base. The most interesting solutions are those for which the source or sink lies above the asymptotic level of the free stream at infinity. As $\varsigma_{B} \rightarrow-\infty$, the source. moves higher and higher above the level of the free stream, and the flow looks more and more like a fountain. It appears unlikely that an equivalent flow of this type could exist for a sink in a real situation, but this is not so clear for a source.

The similarity in shape of these results to those of Vanden-Broeck and Keller [9] for the full nonlinear free-surface condition raises the question of whether the full nonlinear solutions apply for a source, a sink, or for some range of both. Further, the existence of solutions at infinite Froude number indicates that any cusp-like free surface shape calculated at finite Froude number can only be regarded as determining an upper bound for the critical drawdown Froude number, as suggested by Yih [10]. This is supported by the work of VandenBroeck and Keller [9], who found cusp-like free-surface shapes at all values of $F$ greater than some finite value, for all bottom geometries similar to those considered here.

\section{Acknowledgements}

The author would like to thank Prof. J. Imberger and Dr. S. Monismith for their comments on a draft of this paper.

\section{References}

[1] M. Abramowitz and I. A. Stegun (eds.), Handbook of mathematical functions (Dover, New York, 1970)

[2] A. Craya, "Theoretical research on the flow of nonhomogeneous fluids", Houille Blanche 4 (1949), 44-55.

[3] I. L. Collings, "Two infinite Froude number cusped free surface flows due to a submerged line source or sink" J. Austral. Math. Soc. Ser. B 28 (1986), 260-270. 
[4] G. C. Hocking, "Cusp-like free-surface flows due to a submerged source or sink in the presence of a flat or sloping bottom" J. Austral. Math. Soc. Ser. B 26 (1985), 470-486.

[5] D. H. Peregrine, "A line source beneath a free surface", Mathematics Research Centre, Univ. Wisconsin Rep. 1248 (1972).

[6] F. G. Tricomi, Integral Equations (Interscience, 1957).

[7] E. O. Tuck, "Application and solution of Cauchy singular integral equations", in The application and numerical solution of integral equations (ed. R. S. Anderssen et al) (Sijthoff and Noordhoff, 1980)

[8] J. M. Vanden-Broeck, L. W. Schwartz and E. O. Tuck, "Divergent Low-Froude-number series expansion of non-linear free-surface flow problems", Proc. Roy. Soc. London Ser. A 361 (1978), 207-224.

[9] J. M. Vanden-Broeck and J. B. Keller, "Free surface flow due to a sink" J. Fluid Mech. 75 (1987), 109-117.

[10] C. S. Yih, "Effect of geometry on selective withdrawal" (to appear in J. Fluid Mech.) 